\title{
Biomedical Applications of Natural Polymer Based Nanofibrous Scaffolds
}

\author{
Preethi GU, Manu M Joseph, Unnikrishnan BS, Shiji R and Sreelekha TT*
}

Laboratory of Biopharmaceuticals, Division of Cancer Research, Regional Cancer Centre, India

*Corresponding author: Dr. T.T. Sreelekha PhD, Assistant Professor, Division of Cancer Research, Regional Cancer Centre, Trivandrum, Kerala-695011, India, Tel: +91471 2522378, E-mail: ttsreelekha@gmail.com or ttsreelekha@rcc.gov.in

\begin{abstract}
Nanotechnology is one of the most popular branches of science in the current decade where nanofiber scaffolds (NS) attain principal attraction. NS are composed of several nanometer to micrometer diameter fibers similar in nature to collagen, keratin and elastin fibers of the extracellular matrix (ECM) and helps in cell attachment, proliferation and differentiation. NS with desired features can be fabricated using various chemical, physical and biological methods such as electrospinning, phase separation, self-assembly, templating etc. Most often NS are fabricated using ECM derived compounds, naturally occurring polysaccharides such as chitosan, pullulan, dextran and many other synthetic compounds. Like ECM in natural niche NS interacts with cells and hence holds wide applications in Tissue engineering (TE)/ regenerative medicine where they are used as replacement for nerve, blood vessels, skin, muscle, bone and cartilage. They have also established applications in wound healing, drug testing and drug/ growth factor/ gene delivery. Application of cell free scaffolds for the culture of stem cells isolated from individual persons could pave way towards personalised therapy using TE. Cancer cells cultured in 3D microenvironment created with NS were observed to enhance the epithelial to mesenchymal transition. The present review principally focuses on various fabrication techniques and the application of various natural and synthetic compounds in tissue engineering.
\end{abstract}

\section{Keywords}

Nanofiberscaffolds, Electrospinning, Polysaccharides, Tissue engineering, 3D Culture

\section{Introduction}

Nanotechnology is a multi-disciplinary field that aims to create materials and devices that can be applied in aerospace, medicine, information technology, agriculture and energy production. As nanotechnology advances, new fields are explored with advanced technologies to improve the quality of life. With the progress in science and technology, nanoparticle (NP) synthesis has accelerated, with emergence of new methods using various natural products for eco-friendly (green) synthesis of NPs. Plant extracts, microbial cultures and other natural compounds are widely exploited for the fabrication of NPs. Other than NPs one of the emerging fields is based on nanofibers. Synthesis of biogenic nanofibers for scaffold modelling has attracted widespread applications in biomedical field. Nanofibers are fibers of diameter ranging up to 100 to $1000 \mathrm{~nm}$. Interwoven meshes of nanofibers are termed nanofiber scaffolds, which can be random or aligned [1]. Currently nanofiber fabrication is performed using three main techniques: electrospinning, self-assembly and phase separation, of which electrospinning gives scaffolds with most promising TE applications.

Nanofiber scaffolds (NS) are prepared using a few nanometres to micrometre diameter fibers which are interwoven in a specific pattern [2]. The word scaffold literally means a "platform for support" and is explored in nanobiotechnology for the support and growth of cells. Scaffolds will mechanically and structurally support the growth and proliferation of cells grown on them. NS could be fabricated using several techniques of which the most prominent one is electrospinning [3]. Other major procedures for the synthesis of NS include phase separation, self- assembly, templating and melt blowing. NS can be engineered for specific applications by incorporating chemicals/ drugs using biologically active compounds such as polysaccharides and proteins.

Extracellular matrix (ECM) derived components such as collagen, elastin and keratin fibers are mostly used as they naturally possess a fibrous nature and are easy to get fabricated into fibrous scaffold. ECM components are naturally occurring and can be easily manipulated for cell-cell attachment and cell-basement membrane attachment. Proteins, polysaccharides and other biological materials have also evaluated for the fabrication of scaffolds as they possess several unique properties such as water solubility, water absorption, biodegradation, hydrophilicity/ hydrophobicity and biocompatibility. Cellulose, dextran, amylose, chitin, hyaluronic acid, chondroitin sulphate, heparin and glycosaminoglycan are widely used as material for the fabrication of scaffolds [4]. Natural polymers hold many advantages over synthetic ones such as cheap availability, non toxicity, biocompatibility and biodegradability. They also hold certain constrains over synthetic ones including lack of control of molecular weight, chain configuration, polymerization kinetics and versatility in chemistry [5]. Several synthetic compounds such as Poly lactic acid (PLA), Poly glycolic acid (PGA), Poly lactic co glycolic acid (PLGA), Polyhydroxyalkanoates (PHA), Polyvinyl alcohol (PVA), Poly caprolactone (PCL) and bioactive ceramics also play promising roles in the fabrication of scaffolds [6].The three dimensional architecture of NS provides a more mimicking condition of the natural niche for the growth of cells as compared with the conventional $2 \mathrm{D}$ cell culture

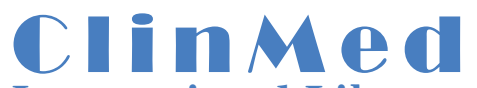

International Library

Citation: Preethi GU, Joseph MM, Unnikrishnan BS, Shiji R, Sreelekha TT (2015) Biomedical Applications of Natural Polymer Based Nanofibrous Scaffolds. Int J Med Nano Res 2:010

Received: August 20, 2015: Accepted: September 14, 2015: Published: September 17, 2015 Copyright: () 2015 Preethi GU. This is an open-access article distributed under the terms of the Creative Commons Attribution License, which permits unrestricted use, distribution, and reproduction in any medium, provided the original author and source are credited. 


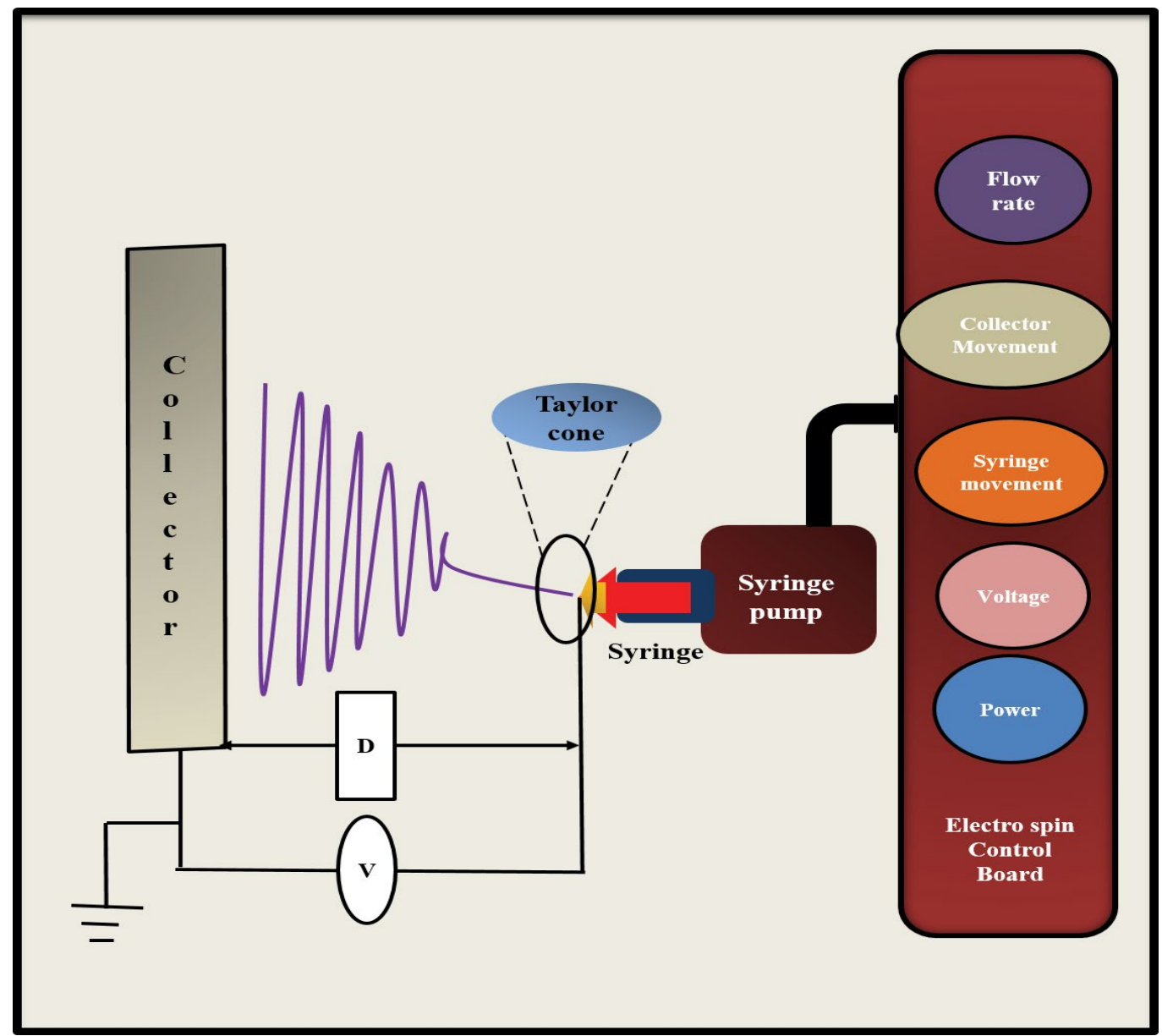

Figure 1: Schematic representation of electrospinning unit: The instrument consists of a syringe connected to a high voltage supply and a collector which is grounded. The control panel contains mainly voltage and flow rate regulators.

methods. Growth of cells in 3D scaffolds found wide applications in tissue engineering, drug delivery and wound healing. Current review mainly focuses on the biomedical applications of nanofibrous scaffolds fabricated using polymers with special emphasis on natural products.

\section{Methods of Nano-Fabrication}

\section{Electrospinning}

Electrospinning is the most popular technique for the fabrication of nanofibers since $18^{\text {th }}$ century, and utilizes electrostatic forces to form fibers from the spinneret. The basic principle behind electrospinning is on application of high voltage, the particles get charged and forms a cone shaped structure called Taylor cone. This leads to the formation of a jet from the tip of Taylor cone. When applied, potential overcomes the surface tension of the solution which moves along the electric field and get collected to the grounded collector (Figure 1). Under predefined processing conditions and continuous feeding of the polymer solution or melt, a uniform jet can be obtained. This is influenced by various parameters such as viscosity and conductivity of the solution, voltage applied, and distance between the needle tips to the collector and flow rate [7]. The new development in electrospinning includes multi-layered electrospinning, core-shelled electrospinning, two-phase electrospinning, blowing-assisted electrospinning and post alignment methods [8].

One of the greatest advantages of electrospinning is the control over the thickness and porosity of the material under fabrication. It has a simple experimental setup and can produce fibers of 50- 100 $\mathrm{nm}$ or greater diameter with accuracy and precision. The nanofibers produced will have high surface area and porosity. Porous structures allow the optimal interaction of the cells entrapped inside the scaffold; small pores reduce the penetration of cells and large pores reduce the cell attachment due to reduced area. Adequate porosity will provide cell in growth, metabolite transport and vascularization [6]. Scanning Electron Microscopy, mercury porosimetry like techniques are used for analysing porosity of the NS. One of the unique advantage of electrospinning from other fabrication techniques is the ability to produce oriented nanofibers [1]. Scaffolds and fibers synthesised using electrospinning find wide commercial applications: as reinforcing fibers in composite materials, non-wetting surface layer on ordinary textiles, polymeric separation membranes, a route to the production of nonwoven fabrics [9] and many promising biomedical applications.

Electrospinning was used for the production of ultrafine gelatin fibers by Zhang et al. [10] wherein along with the gelatin, they have tried PCL/ gelatin mixture also which was further investigated as matrix for bone marrow stromal cell culture. The scaffolds not only favoured the surface attachment but also the migration of the cells up to $114 \mu \mathrm{m}$ inside the scaffold. In a similar type of work, cell adhesive peptides, modified alginate and unmodified alginate together with poly ethylene oxide was electrospun to obtain fibers of 150 to $250 \mathrm{~nm}$ size which support the growth of fibroblast [11]. Chitin is a polymer or $\mathrm{N}$-acetyl glucosamine and its deacetylated form is chitosan. Both chitin and chitosan found diverse applications as starting materials for the fabrication of various scaffolds with potent biomedical uses. Chitin nanofibers were produced by the electrospinning of the extract solution of shrimp shell [12].

Micro or nanofibers were prepared by electrospinning using a blend of chitosan and PCL, where trifluroacetic acid was used as co-solvent and the resultant fibers found wide applications in TE [13].Wet spinning of chitosan was done to develop nanofibers using sodium hydroxide or sodium tripolyphosphate [14]. The potential of chitosan-PCL and PCL fibrous mats as skin tissue engineering scaffolds were assessed by cell adhesion, viability, proliferation and act in distribution using human keratinocytes (HaCaT) and L-929 


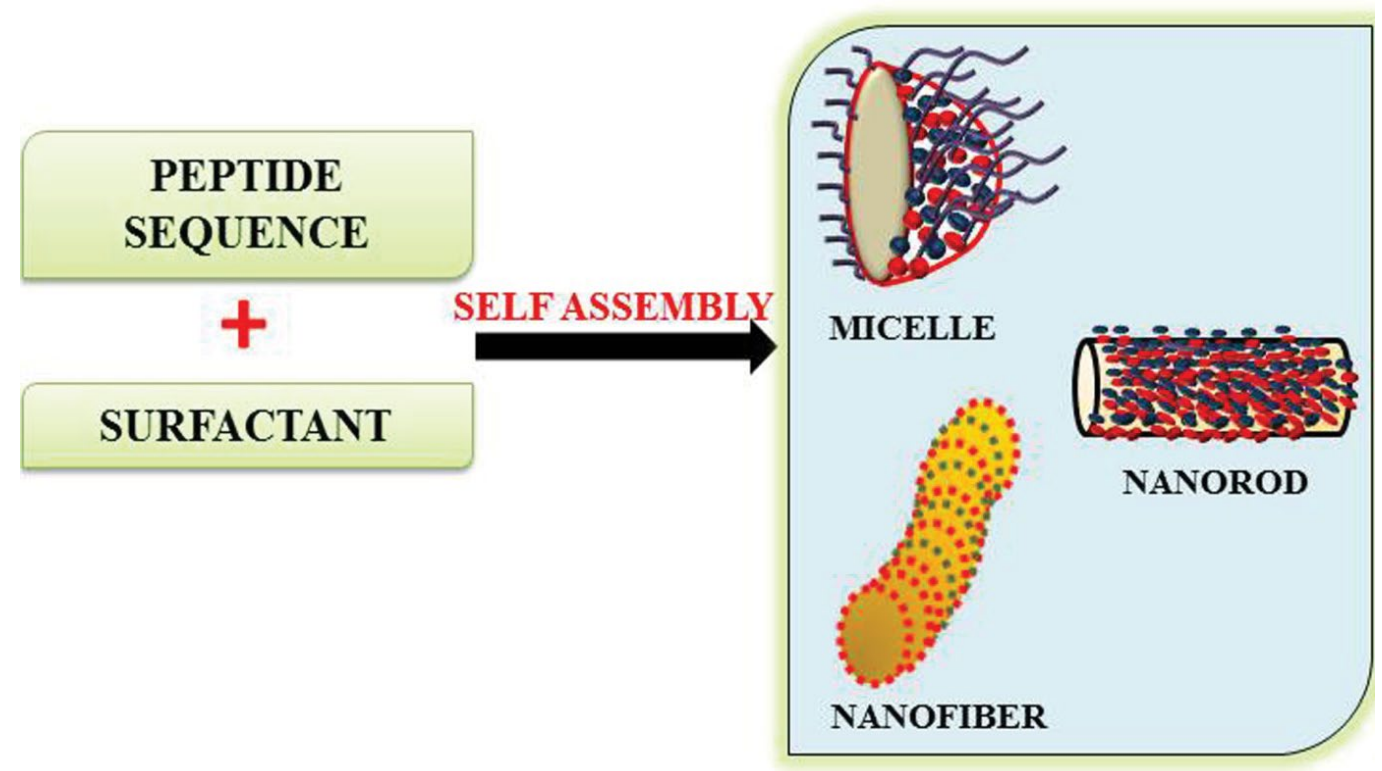

Figure 2: Schematic illustration of self-assembled nanostructures by peptides with surfactants. A peptide sequence in the presence of a detergent such as fatty acids forms different structures like nanofibers, nanorods or micelles by varying the parameters.

cell lines. Chitosan- PCL is a better scaffold for attachment and proliferation of keratinocytes and is a potential material for skin tissue engineering. Another work reported chitosan blended with PLA and polyoxyethylenenonylphenol ether to improve the electrospinnablity of chitosan. Cytotoxocity tests showed that CS/PLA/TX-15 nanofiber mats were safe and nontoxic toward Human Mesenchymal Stem Cells (hMSCs) [15]. Composite polysaccharide nanofibers containing chitosan and hyaluronic acid was prepared by electrospinning and characterized by Maeda et al. [16] which found applications in wound healing and drug delivery. The first reported study on extra cellular polymeric substance of cyanobacterial origin (EPS) and PVA blend nanofibers embedded onto a poly vinyl fluoride (PVDF) membrane is studied for the application in water filtration [17]. Polyaniline PVA and chitosan blend nanofibers were prepared by electrospinning [18]. Vascular Endothelial Growth Factor (VEGF) loaded poly fucose- pullulan- dextran electrospun nanofibers were fabricated and characterized by Rujitanaroj et al. [19]. Subcutaneous implantation of the scaffold in mice showed enhanced angiogenesis. Amranth protein (API) and pullulan composite fibers prepared by electrospinning demonstrated that the ability to generate encapsulation structures from API depends, not only on the protein conformation, but also on the solution properties (conductivity, surface tension and viscosity) [20].

\section{Self- assembly}

Self-assembly is a bottom up approach for the fabrication of NS wherein spontaneous organization of molecules into a structurally arranged form through pre-programmed noncovalent interactions occurs. It is an easy method for the preparation of injectable scaffolds and to encapsulate inside the hydrogels but the pore size and shape of the formed scaffold is difficult to control. The process includes the following steps: polymer dissolution, liquid-liquid phase separation and gelation, solvent extraction, freezing and freeze-drying [21]. It gives a polymer rich and lean phases which are interconnected forming scaffold architecture.

A novel alginate microfiber on bead was reported by Park et al. [22], wherein alginate beads were produced by calcium chloride precipitation. The beads were then kept in culture medium which induced microfibers of $10 \mathrm{~mm}$ in length and was used as 3D scaffold for cell culture. The rigid chain of chitosan was used for the selfassembly of silk fibroin which promotes the formation of 3D scaffolds. This scaffold facilitates the attachment of hepatocyte in a better way as compared to PLGA scaffolds [23]. Chitosan NS was widely reported to be produced through self-assembly process. A modified water soluble form of chitosan, $\mathrm{N}$-maleoyl chitosan formed self-assembled fibers with a diameter of $2.5 \mu \mathrm{m}$ and length of $100 \mu \mathrm{m}$ [24]. Peptides can be self-assembled form different structures such as nanotubes, nanofibrous scaffolds, helical ribbons, micelles etc. through voluminous methods (Figure 2). Nanofibers could be easily produced through self-assembly by fluctuating the $\mathrm{pH}$. $\mathrm{pH}$ induced self-assembly was used for the synthesis of peptide amphiphile (PA) nanofibers which was able to direct mineralization of hydroxyapatite crystals. Physiological $\mathrm{pH}$ can also induce the formation nanofibers from oppositely charged PAs [25]. Lu and co-workers used collagen and silk for the fabrication of NS and porous scaffolds using the process of self-assembly through water annealing and lyophilisation. Water annealing was used to fabricate insoluble silk based scaffolds and avoiding the use of organic solvents. Nanofibrils ranging 20$100 \mathrm{~nm}$ was obtained, which was similar to native collagen [26]. The advantage of self- assembly process is that they produce nanofibers which are very similar to collagen fibers and enables the simultaneous production of macro and nano structures. It is a simple procedure and the requirements for the process are very minimum [1].

\section{Phase separation}

Phase separation is a thermodynamic process in which a homogeneous multi-component system tends to separate into multiple phases to lower the system free energy, initiated thermally or by using non solvent, forming a polymer rich and lean phase. Polymer rich phase solidifies and polymer lean phase becomes the voids in between (Figure 3). Synthetic and natural biodegradable materials such as PLA, PLGA, PCL, Polyurethane (PU), Poly ether ester urethane (PEEU), collagen, gelatin and chitosan have been used in the fabrication of scaffolds. In order to obtain various structures of NS prepared via Thermally Induced Phase Separation (TIPS) process, researches have made several modifications to the process and combined it with other processes such as porogen leaching and electrospinning.

Liu et al. [27] obtained gelatin fiber with diameter ranged from $50 \mathrm{~nm}$ to $500 \mathrm{~nm}$, which is on the same size as natural collagen fibers where the average fiber diameter did not change with different gelatin concentrations. The scaffolds prepared with $7.5 \%(\mathrm{w} / \mathrm{v})$ gelatin solution had a low density, $97.51 \%$ of porosity and well defined macro pores with pore size of $250-420 \mu \mathrm{m}$. In a similar work, Qu et al. developed an approach for the preparation of three-dimensional (3D) nano-fibrous gelatin/silica bioactive glass (NF-gelatin/SBG) 

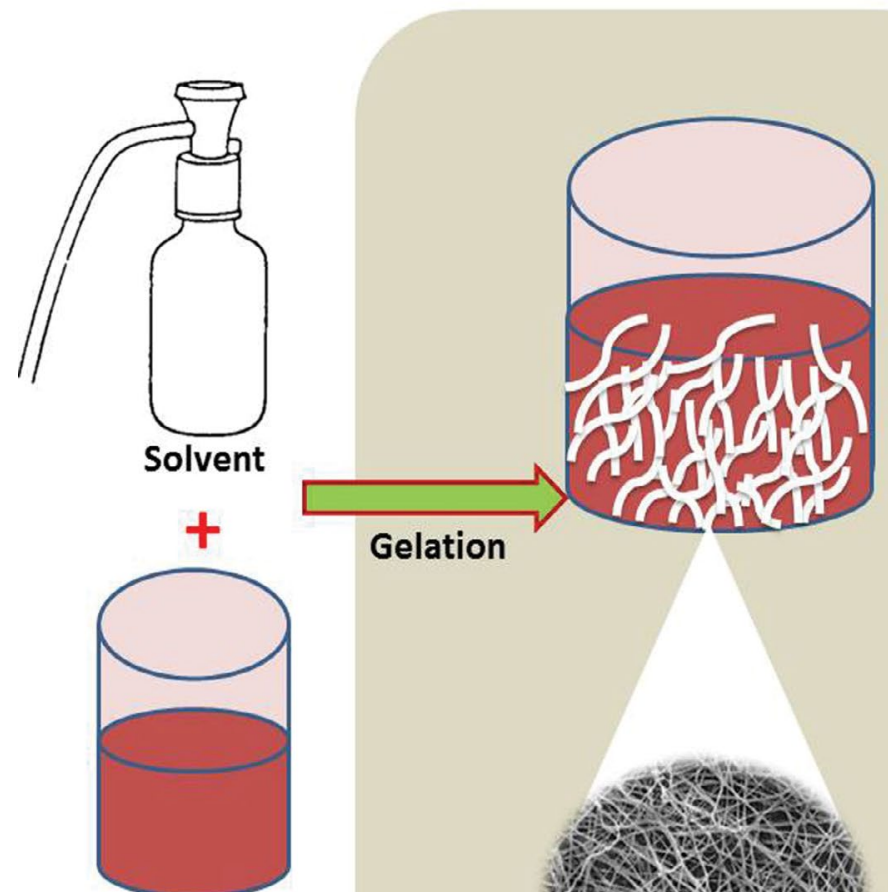

Polymer

\section{Nanofibre}

Figure 3: The figure depicts phase separation process upon addition of a solvent to a polymer solution. Polymer rich and lean phases are separated by selfinduced gelation and the formation of porous matrix made of nanofibers.

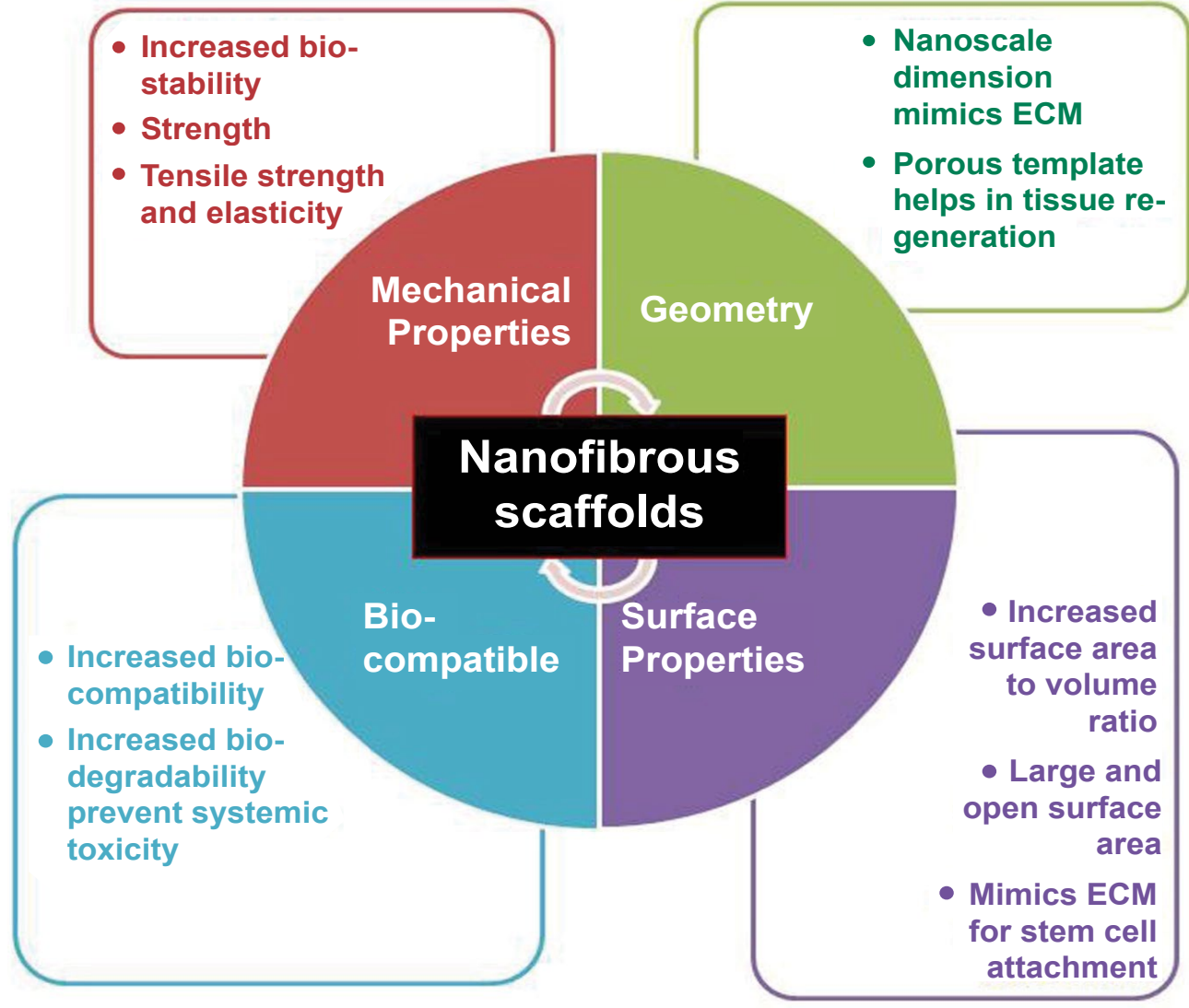

Figure 4: Schematic representation of properties of nanofibrous scaffold which includes geometry, mechanical competence, bio-compatibility and degradability and surface properties. 
hybrid scaffolds. The prepared hybrid NS mimic the nano-structured architecture and chemical composition of natural dental ECM. This fabrication process involved the combination of a thermally induced phase separation, sol-gel and porogen leaching process. The careful engineering process generated NS with natural ECM-like architecture, porosity, well-defined pore size, inter connectivity with improved mechanical strength. These scaffolds favour the growth of human dental pulp stem cells (DPSCs) in cell culture study with a significantly higher proliferation rate as compared to NF-gelatin scaffolds under the same conditions [28].

\section{Properties}

In order to fabricate a NS of desired applications it should possess certain features which are described below (Figure 4).

\section{Geometry}

Macro and microstructure and interconnectivity are the external geometric features which provide the surface for cell attachment. Nano to macroscale structure geometrically or topologically mimics the native state of ECM in living tissues. 3D scaffolds are capable of regenerating tissue and organs in their normal physiological form. Average pore size, size distribution, volume, interconnectivity, shape, throat size and wall roughness are important factors to consider while designing a scaffold [29]. It delivers a porous biocompatible network into which the surrounding tissue is induced and acts as a temporary template for the new tissue's growth and restructuring.

\section{Surface properties}

Surface energy, chemistry, surface area and charge are included in the surface properties of a scaffold [30]. Scaffolds with a large and open surface area are favourable for cell attachment. For example, a high internal surface area-to-volume ratio is essential to house the number of cells required to replace or restore tissue and organ functions. The surface properties can be selectively modified to enhance the performance of the biomaterials. For instance, by altering the surface functionality using thin film deposition, optimal surface properties can be attained [31]. Hence, surface modification of biomaterials is becoming an increasingly popular method to improve device multifunctionality and mechanical properties to suite biomedical applications.

\section{Biocompatibility and biodegradability}

Biocompatibility, in broad terms refers to the ability of a biomaterial to perform its desired function; with respect to medical therapy, without eliciting any undesirable local or systemic effects. With reference to NS, biocompatibility refers to the capability to perform as a substrate that will support the appropriate cellular activity, including the facilitation of molecular and mechanical signalling systems [32]. Scaffold degradation can occur through mechanisms that involve physical or chemical and/or biological processes that are mediated by biological agents, such as enzymes in tissue remodelling. The biodegradable scaffold gradually degrades by predetermined period to be replaced by newly grown tissue from the adhered cells [33].

\section{Mechanical competence}

Various physical properties of a biomaterial, shaping its mechanical competence, play a vital role for a biomaterial to be used in a tissue engineering application and are very critical to the success of the implant [34]. Bio-stability of many scaffolds is determined by the factors such as strength, elasticity, absorption at the material interface and its chemical degradation. The NS should have proper mechanical properties and degradation rate with the bioactive surface to encourage the rapid regeneration of the tissue [35]. Polysaccharides represent an ideal material with all the above mentioned features for their use in fabrication of biocompatible scaffolds. Fortunately, the options for tissue engineering expanded by the use of fibrous proteins, which are now widely exploited for NS preparation due to its mechanical integrity and stability [1].

\section{Properties in relation to the nanofibrous structure}

The properties of the nanofibers explained above may change with accordance to the application of the NS. Every work dealing with the application goes through a characterization part which includes the physical and mechanical properties of the NS. Nanofibers are better drug delivery systems due to the high specific surface area and short diffusion passage length. The drug release rate can be finely controlled by modulation of nanofiber morphology, porosity and composition [36]. 3D nanofibrous scaffolds are characterized by high porosity with a wide distribution of pore diameter, high surface area-tovolume ratio, and morphologic similarities to natural collagen fibrils. Mechanical stability to provide a carrier for MSC transplantation [24]. It is assumed that the large surface area of nanofibers with specific surface chemistry enables attachment of cells and control of their cellular functions. Increasing roughness of the surface increases the endothelialisation of biomaterials [37]. Enzyme immobilization, filtration is done using chitosan PVA blend nanofibers due to their biocompatibility, high surface/volume ratio and large porosity [36].

\section{Applications}

\section{Tissue engineering}

Tissue engineering (TE) is the use of a combination of cells, engineering and materials, methods and suitable biochemical and physico-chemical factors, to improve or replace biological functions. ECM analogues are usually best suited for TE applications. Stem cells can be delivered to appropriate sites in the body, by injection or by implantation of the NS. Xylan along with PVA is electrospun to give nanofibers, which were found to be suitable materials for skin regeneration. The cell-biomaterial interactions are studied by culturing human foreskin fibroblasts on Xylan/ PVA Glutaraldehyde (Glu) vapor cross-linked and Xylan/PVA/Glu blend NS which have good potential for fibroblast adhesion, proliferation and cell matrix interactions relevant for skin tissue regeneration [38]. In a different study, $\mathrm{Xu}$ and co-workers fabricated vascular stents from poly (lactide- $\varepsilon$-caprolactone)/collagen/nano-hydroxyapatite (PLCL/Col/nHA) by electrospinning and the anti-clotting properties of stents were evaluated for anticoagulation surfaces modified by the electrostatic layer-by-layer self-assembly technique [39]. Gelatin blended with polyglycolic acid (PGA) in different ratios were electrospun to produce nanofibers which were analysed for biocompatibility using human umbilical vein endothelial cells and human umbilical artery smooth muscle cells. The study suggested that tubular scaffolds with an inner layer of PGA/10 wt\% gelatin and an outer layer of PGA/30 wt\% gelatin are promising scaffolds for vascular tissue engineering [34]. Wang et al. reported heparin-loaded gelatin fibrous scaffolds fabricated via electrospinning technology crosslinked using glutaraldehyde. Gelatin-heparin scaffolds are promising candidates as a drug delivery system for the localized administration of heparin to the site of vascular grafts [40]. In a separate study, a hydrophilic and compliant polyurethane namely Tecophilic (TP) blended with gelatin (gel) at a weight ratio of 70:30 was electrospun to fabricate a tubular composite scaffold with biomechanical properties closely simulating those of native blood vessels. The scaffold greatly improved the capacity of the scaffold to serve as an adhesive substrate for vascular smooth muscle cells (SMCs) [41]. In another study, nanofibers of PLGA and gelatin were electrospun and the effect of parameters namely polymer concentration, acid concentration, flow rate and voltage on the morphology of the fibers were investigated. The results indicated desirable bioactivity and hemostasis of the scaffolds with the capability of encapsulation and controlled release of the protein, which can be served as skin tissue engineering scaffolds and wound dressings [42]. Chitosan and PVA blend were used for the formation of nanofibers using electrospinning, and the products found application as dermal substitutes. The in vitro evaluation was done using 3T3 fibroblast cell lines and in vivo study was done along with growth factor R Spondin1 [43]. The optimum condition for gelatin/ GAG electrospinning was also introduced using the model obtained in one study. The potential use of optimized electrospun mat in skin tissue engineering was evaluated using culturing of human dermal 
fibroblast cells (HDF). The electrospun gelatin / GAG nano fibrous mats have a potential for using as scaffold for skin, cartilage and cornea tissue engineering [44]. Aloe vera, silk fibroin and hydroxyapatite incorporated scaffolds prepared using electrospinning was found to increase osteogenesis in vitro. The scaffolds showed significant increase in cell proliferation, osteogenic differentiation, osteocalcin expression and mineral deposition [45]. Srivastava et al. reported the enhanced osteoblast differentiation of mouse embryonic cells on a novel nanofiber matrix of polygalacturonic acid and gelatin wherein 3T3 L1 cells were used to analyse the cytocompatibility of the scaffold [46]. A study identified the biopolymers - Aloe vera, silk fibroin and curcumin loaded into polycaprolactone (PCL) as suitable substrates for tissue engineering. The average fiber diameter of obtained fibers ranged from $250 \mathrm{~nm}$ to $350 \mathrm{~nm}$ and the incorporation of biopolymers decreased the hydrophobicity of the scaffolds [47].

\section{Wound healing}

For a scaffold to be used in wound healing applications, it demands some special properties such antimicrobial properties or hemostatic activities and hence the scaffold material should be carefully chosen. Chitosan has both antibacterial and hemostatic properties and is an ideal material to be used for the preparation of scaffolds meant for wound healing applications. Bioactive dressings should fulfil several criteria like biocompatibility in the absence of cytotoxicity, easily applied to the wound, able to conform to the wound surface, easily removable after healing and bio-functionalized to allow the slow release of bioactive agents.
Engineering skin substitutes by tissue engineering approach relies upon the creation of 3D scaffolds as ECM equivalent to guide cell adhesion, growth and differentiation to form a functional and structural skin tissue. The 3D scaffolds not only aid in wound dressing, but also can provide support both for dermal fibroblasts and the overlying keratinocytes for skin tissue engineering. Collagen is the most important dermal replacer used in various forms such as electrospinning to porous scaffolds. Electrospinning of type I collagen using hexafluro isopropanol (HFIP) yielded nanofibers in the size range of $460 \mathrm{~nm}$, where the cross linked collagen fibers accelerate early wound healing response [48]. Gelatin, the degradation product of collagen also was extensively used for fabrication of nanofibers using electrospinning and produced a similar response as observed with collagen. An artificial dermal layer produced by combining gelatin and PCL thorough electrospinning fibers of gelatin onto the PCL matrix (Tegaderm ${ }^{\mathrm{TM}}$ ) was found to support fibroblast adhesion and proliferation [49]. Gelatin, in combination with polyurethane was evaluated for its potential in wound healing by Kim et al. [50]. Gomes and co-workers compared the cell-scaffold interaction and wound healing promotion performance of electrospun nanofiber mats from three polymers, namely, a polyester (PCL), a protein (gelatin from cold water fish skin, GEL) and a polysaccharide (chitosan). However, amiable cell growth was only observed in the PCL and chitosan scaffolds. In in vivo tests chitosan scaffolds had the highest impact on the healing process by decreasing the extent of wound contraction and enhancing the production of a neodermis and re-epithelialization of the wound [51]. Schneider et al. [52] used self-assembled peptide nanofibers along with EGF for the dermal reconstitution and wound

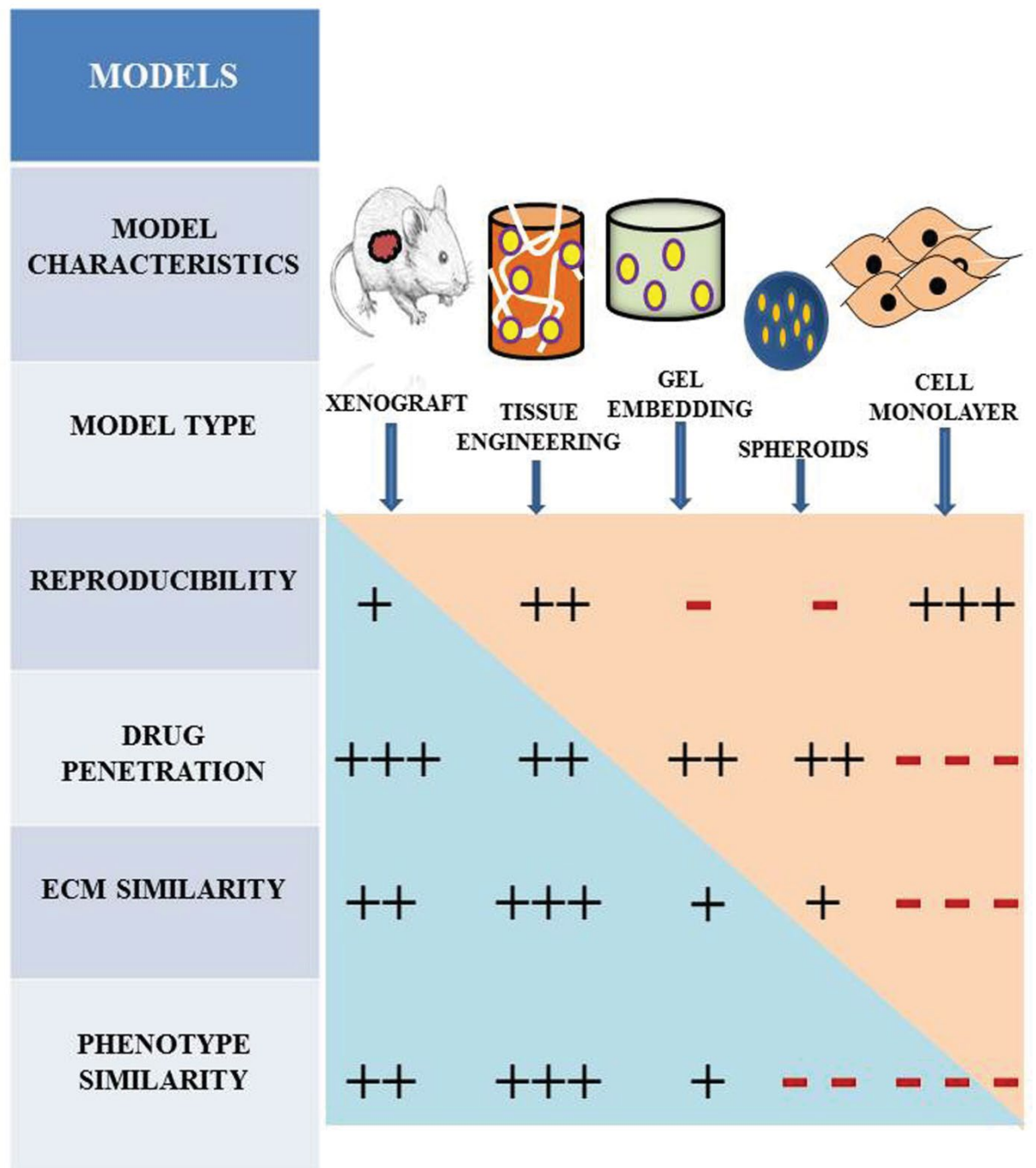

Figure 5: Schematic representation of different tumor models and their main characteristics compared with each other. Xenograft and 3D scaffolds used for tissue engineering have comparable results. All of the models represented here have great advantage over 2D monolayer culture. 
re-epithelialisation. The study uncovered that the scaffold could cover the wound 5 times more than the negative control by localized growth factor release and activation of wound repair mechanisms. Gellan based wound healing nanofibers was reported for the first time by Vashisth et al. [53]. Gellan based nanofibers of $20 \mathrm{~nm}$ in size was synthesized by mixing it with a polymer PVA and electrospun at specific conditions. Human dermal fibroblasts were cultured to analyse the biocompatibility of the scaffold and as an evidence for wound healing application.

\section{Drug testing and 3D culture}

In order to evaluate the efficacy and efficiency of drugs, treatment with cells both in vitro and in vivo is a mandatory step. Conventional 2D cell cultures or animal models are usually used but accompanied with several drawbacks, the main one being that they don't mimic the environment in natural systems and so the efficacy of drug tested in in vitro $2 \mathrm{D}$ cultures may not produce the same effect when evaluated in animal models or in human trials. Literature revealed that the genomic expression profile of the cells in the $3 \mathrm{D}$ culture is quite different from the cells in 2D. Kim et al. [54] proposed that 3D cultures provide similar conditions for a cell and drug to what is observed in endogenous environment. These aspects can be cleared out up to a larger extend by replacing $2 \mathrm{D}$ cultures with $3 \mathrm{D}$ NS, which mimic the in vivo cellular microenvironment. 3D NS play an intermediate between the conventional 2D cultures and animal models. The most common 3D culture systems are scaffolds and hydrogels wherein the interconnected pores and fibers help in cell migration and growth. 3D fiber deposition and electrospinning was used by Moroni et al. [55] to fabricate scaffolds for the enhancement of cartilage tissue formation, where the 3D fiber deposition provided structural integrity and electrospinning gives cell entrapment ability to the scaffold. Electrospinning of collagen peptide and PHBV were tested for the 3D culture of gastric cancer cells. Chemosensitivity assays done on these scaffolds work by directly analyzing effects of therapies on living cancer cells to find out potential anticancer drugs with respect to each individual type of tumors. Co-culturing of cells in a 3D microenvironment are widely used to study the effect of drug to the surroundings, especially in anticancer drug testing; it is very important to study the effect of drug to the surrounding cells as it may inhibit or kill the normal cells also. In 3D co-culturing techniques, diverse types of cells can be grown together and the specific biological effects can be properly evaluated, which will mimic the in vivo conditions. Figure 5 gives an overview comparison of in vitro 2D culture, $3 \mathrm{D}$ culture and in vivo drug testing models.

\section{Delivery of drug and other entities}

Sustained and site specific delivery of biogenic materials such as growth factors, genes or drugs is very important for efficient treatment of diseased conditions. Various surface modification techniques, as depicted in Figure 6 can be done for engineering the NS for specific biomedical applications. Plasma treatment, commonly used to tailor surface adhesion and wetting properties to the polymer by changing surface chemical composition was found to be an effective treatment strategy. In order to create new surface functionalities, wet chemical method was used which involved the use of acidic or basic media. Also, to conquer hydrophilicity and addition of functional groups, surface graft polymerization technique could also be employed. Surface modification can also be achieved

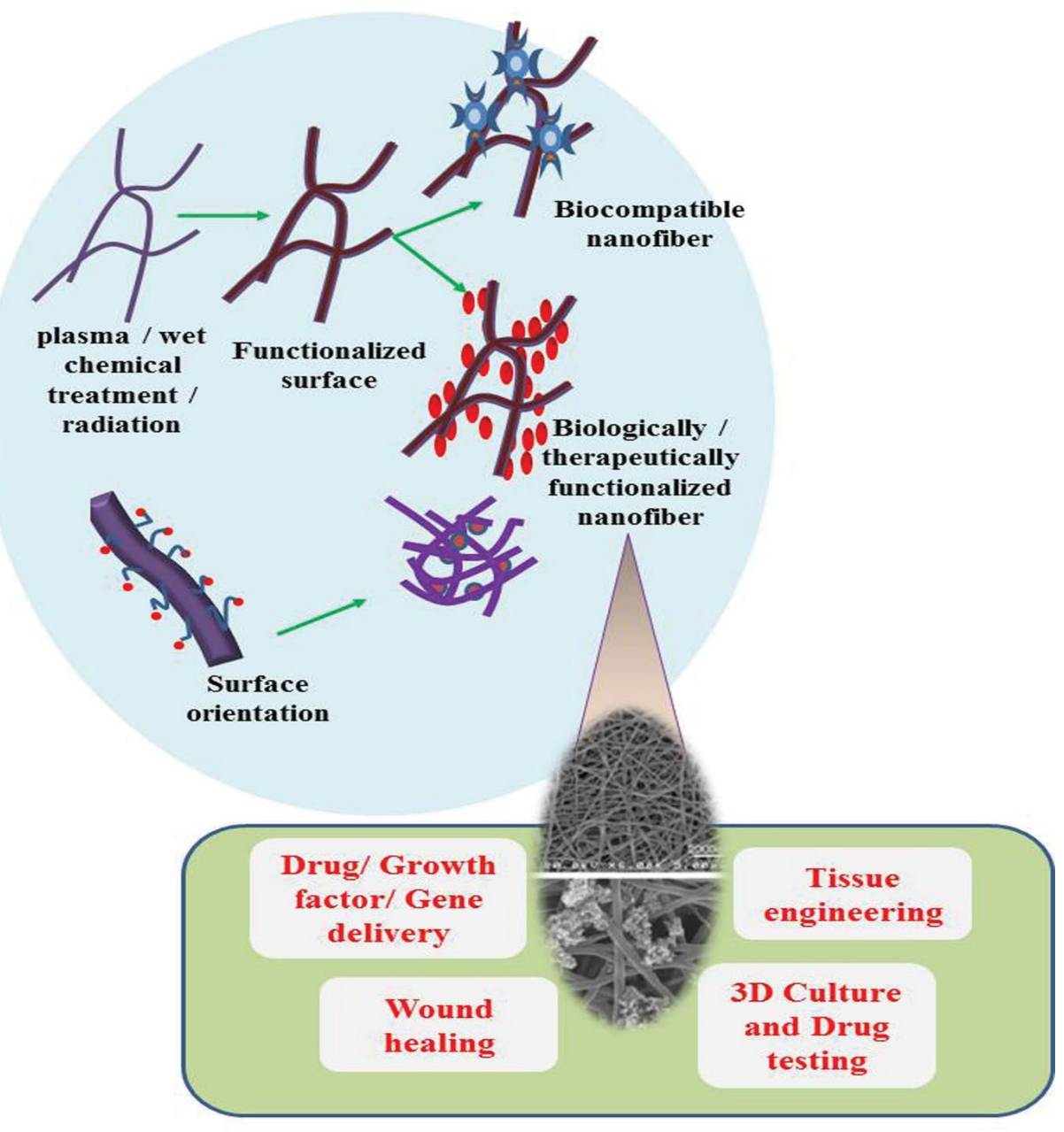

Figure 6: Surface modification of electrospun fibers and their applications. Radiation, wet or chemical treatments are used for the functionalization of nanofibers and different moieties can be tagged such as bioactive compounds or known drugs. Surface orientation of the nanofibers can be aligned or random and molecules can also be embedded within the fiber. Chemical or physical embedding can be done for functionalization. The functionalised fibres found promising applications in TE, 3D culture, drug delivery, wound healing and much more. 
using co-electrospinning wherein for the efficient entrapment of drug two kinds of fabrication techniques are used. One uses drug mixed with the polymer solution while the other uses scaffolds entrapped with drugs or nanoparticle formulations after the fabrication of scaffolds [56]. A variety of bioactive molecules such as anti-cancer drugs, enzymes, cytokines, antibiotics and polysaccharides are entrapped within the interior or physically immobilized on the surface for controlled delivery. Sustained release and encapsulation of Mefoxin ${ }^{\circledR}$, a hydrophilic antibiotic was studied by Kim et al. [57], where the antimicrobial activity against Staphylococcus aureus was tested and was observed to inhibit $90 \%$ of the bacterial growth and hence proved to be an effective platform in post-surgical adhesions and infections. Silk fibroin scaffolds containing bone morphogenetic protein 2 (BMP 2) and nanoparticles of hydroxy apatite (nHAP) were used for in vitro bone formation from mesenchymal stem cells. The coexistence of BMP-2 and nHAP in the electrospun silk fibroin fibers resulted in the highest calcium deposition and up regulation of BMP-2 transcript levels when compared with the other systems. These results suggested the potential role of electrospun silk- fibroinbased scaffolds in bone tissue engineering [58]. Bone-mimetic electrospun scaffolds composed of polycaprolactone, collagen I and nanohydroxyapatite (PCL/col/HA) supported greater MSC adhesion, proliferation and activation of integrin related signalling cascades. Successful and active drug delivery indicated that the adsorption of the factors into the scaffolds doesn't affect the activity of the growth factor [59]. Gene-activated matrix (GAM) using cellular scaffolds are used for gene delivery [60]. Lim et al. employed a chitin/alginate fibrous matrix system to provide a better spatial control for plasmid localization. The nonwoven fibers were injected with cells by needle punching method for transgene expression in the cells [61].

Scaffolds are used in a wide variety of systems for drug delivery. Most common example is in topical or implanted systems. Wound dressings are one of the specific applications in these types of systems. Electrospun nanofibers show capability for wound dressing due to their characteristics, such as extremely high surface area, efficiency in absorbing exudates and adjusting the moisture content in the wounds. High porosity gives air circulation and small pore size reduces infections [62]. Epidermal growth factor loaded silk fiber has demonstrated the application toward wound healing and enhanced wound closure [63]. Cancer therapy is another area which requires the scaffolds for drug delivery due to poor solubility and instability of drugs used, low efficacy in solid tumors and side effects. In order to achieve a prolonged period of function, maximum efficiency, and minimum side effects to health, methods such as local and sustained postsurgical drug delivery have been explored using NS. The researchers have loaded paclitaxel in chitosan nanofibers coated with hyaluronic acid and cell proliferation was inhibited with low quantities of drug [64].

\section{Conclusion}

Nanotechnology provides outstanding advances where, it can offer more solutions to technological problems than any other conventional system. Advances in nano medicine offer new opportunities to improve every aspect of disease management right from early diagnosis to targeted therapy. Nanofiber Scaffolds are a unique class of materials, which can be synthesized from inorganic, polymeric or biological building blocks. Biocompatible threedimensional (3-D) porous scaffolds are of great interest for TE applications and open a bright new avenue for every field of science, especially medical sciences. Biogenic synthesis involves the application of natural polysaccharides and other products for fabricating NS which improves the biodegradability and biocompatibility. Various studies reported provide evidences for wide range applications of NS in tissue engineering and other related fields. Electrospinning technique possesses tremendous scope for engineering biocompatible nanomaterials with advanced properties by using suitable biomaterials and controlling fabrication parameters. Progress in the fabrication of multi-functional NS, holds great promise for soft tissue engineering for repair and/or replacement of damaged or diseased tissues. Many other applications are also there such in development of 3D cultures, drug screening using the cultures, drug/ gene/ cell delivery and so much of relevance stem cell technology. Polymeric nanofibers can be produced using many established methods wherein the fiber properties can be tailored to the intended application, thereby making NS fit for a wide spectrum of biomedical applications.

\section{References}

1. Vasita R, Katti DS (2006) Nanofibers and their applications in tissue engineering. Int J Nanomedicine 1: 15-30.

2. Gupta KC, Haider A, Choi YR, Kang IK (2014) Nanofibrous scaffolds in biomedical applications. Biomater Res 18: 5.

3. Agarwal S, Wendorff HJ, and Greiner A (2008) Use of electrospinning technique for biomedical applications. Polym 49: 5603-5621.

4. Zhao W, Liu W, Li J, Lin X, Wang Y (2015) Preparation of animal polysaccharides nanofibers by electrospinning and their potential biomedical applications. J Biomed Mater Res A 103: 807-818.

5. Venugopal JR, Sridhar S, and Ramakrishna S (2014) Electrospun plantderived natural biomaterials for Tissue engineering. PST 1: 151-154.

6. Garg T, Singh O, Arora S, Murthy R (2012) Scaffold: a novel carrier for cell and drug delivery. Crit Rev Ther Drug Carrier Syst 29: 1-63.

7. Dhanalakshmi M, Lele AK, and Jog JP (2015) Electrospinning of Nylon11: Effect of processing parameters on morphology and microstructure. Mater Today Comm 3: 141-148.

8. Liang D, Hsiao BS, Chu B (2007) Functional electrospun nanofibrous scaffolds for biomedical applications. Adv Drug Deliv Rev 59: 1392-1412.

9. Doshi J, and Reneker DH (1993) Electrospinning Process and Applications of ElectrospunFibers. IEEE conference proceedings 1698-1703.

10. Zhang Y, Ouyang H, Lim CT, Ramakrishna S, Huang ZM (2005) Electrospinning of gelatin fibers and gelatin/PCL composite fibrous scaffolds. J Biomed Mater Res B Appl Biomater 72: 156-165

11. Jeong SI, Krebs MD, Bonino CA, Khan SA, Alsberg E (2010) Electrospun alginate nanofibers with controlled cell adhesion for tissue engineering Macromol Biosci 10: 934-943.

12. Barber PS, Griggs CS, Bonner JR, Rogers RD (2013) Electrospinning of chitin nanofibers directly from an ionic liquid extract of shrimp shells. Green Chem 1-7.

13. Prasad T, Shabeena EA, Vinod D, Kumary TV, Anil Kumar PR (2015) Characterization and in vitro evaluation of electrospun chitosan polycaprolactone blend fibrous mat for skin tissue engineering. J Mater Sci Mater Med 26: 5352.

14. Pati F, Adhikari B, and Dhara S (2011) Development of Ultrafine Chitosan FibersThrough Modified Wet spinning Technique. J Appl Polym Sci 121: 1550-1557

15. Xu J, Zhang J, Gao W, Liang $\mathrm{H}$, Wang $\mathrm{H}$, et al. (2009) Preparation of chitosan/ PLA blend micro/nanofibers by electrospinning. Mater Lett 63: 658-660.

16. Maeda N, Miao J, Simmons TJ, Dordick JS, Linhardt RJ (2014) Composite polysaccharide fibers prepared by electrospinning and coating. Carbohydr Polym 102: 950-955.

17. Santos C, Silva CJ, Büttel Z, Guimarães R, Pereira SB, et al. (2014) Preparation and characterization of polysaccharides/PVA blend nanofibrous membranes by electrospinning method. Carbohydr Polym 99: 584-592.

18. Karim MR (2013) Fabrication of electrospun aligned nanofibers from conducting polyaniline copolymer/polyvinyl alcohol/chitosan oligosaccharide in aqueous solutions. Synth Met 178: 34-37.

19. Rujitanaroj P, Rachida A, Yian SC, Catherine LV (2014) Polysaccharide electrospunfibers with sulfated poly (fucose) promote endothelial cell migration and VEGF-mediated angiogenesis. Biomater Sci 2: 843-852.

20. Aceituno- Medinaa M, Mendoza S, Lagaron JM, Rubio AL (2013) Development and characterization of food-grade electrospunfibers from amaranth protein and pullulan blends. Food Res Int 54: 667-674.

21. Niece KL, Hartgerink JD, Donners JJ, Stupp SI (2003) Self-assembly combining two bioactive peptide-amphiphile molecules into nanofibers by electrostatic attraction. J Am Chem Soc 125: 7146-7147.

22. Park JH, Shin US, and Kim HW (2011) Alginate-Microfibers Produced by Self-Assembly in Cell Culture Medium. Bull. Korean Chem. Soc 32: 431-433.

23. She Z, Liu W, Feng Q (2009) Self-assembly model, hepatocytes attachment and inflammatory response for silk fibroin/chitosan scaffolds. Biomed Mater 4: 045014.

24. Zhu A, Lu Y, Pan Y, Dai S, Wu H (2010) Self-assembly of N-maleoylchitosan in aqueous media. Colloids Surf B Biointerfaces 76: 221-225.

25. Hartgerink JD, Beniash E, Stupp SI (2001) Self-assembly and mineralization of peptide-amphiphile nanofibers. Science 294: 1684-1688. 
26. Lu Q, Wang X, Lu S, Li M, Kaplan DL, et al. (2011) Nanofibrous architecture of silk fibroin scaffolds prepared with a mild self-assembly process. Biomaterials 32: 1059-1067.

27. Liu X, Ma PX (2009) Phase separation, pore structure, and properties of nanofibrous gelatin scaffolds. Biomaterials 30: 4094-4103.

28. Qu T, Liu X (2013) Nano-Structured Gelatin/Bioactive Glass Hybrid Scaffolds for the Enhancement of Odontogenic Differentiation of Human Dental Pulp Stem Cells. J Mater Chem B Mater Biol Med 1: 4764-4772.

29. He L, Liu B, Xipeng G, Xie G, Liao S, et al. (2009) Microstructure and properties of nano-fibrous PCL-b-PLLA scaffolds for cartilage tissue engineering. Eur Cell Mater 18: 63-74.

30. Park K, Ju YM, Son JS, Ahn KD, Han DK (2007) Surface modification of biodegradable electrospun nanofiber scaffolds and their interaction with fibroblasts. J Biomater Sci Polym Ed 18: 369-382.

31. Prabhakaran MP, Venugopal J, Chan CK, Ramakrishna S (2008) Surface modified electrospun nanofibrous scaffolds for nerve tissue engineering. Nanotechnology 19: 455102.

32. Williams DF (2008) On the mechanisms of biocompatibility. Biomaterials 29 : 2941-2953.

33. Langer R, Vacanti JP (1993) Tissue engineering. Science 260: 920-926

34. Hajiali H, Shahgasempour S, Naimi-Jamal MR, Peirovi H (2011) Electrospun PGA/gelatin nanofibrous scaffolds and their potential application in vascula tissue engineering. Int J Nanomedicine 6: 2133-2141.

35. Nair LS, Laurencin CT (2007) Biodegradable polymers as biomaterials. ProgPolymSci 32: 762-798

36. Sun K, Li ZH (2011) Preparations, properties and applications of chitosan based nanofibers fabricated by electrospinning. Express PolymLett 5: 342 361

37. Venugopal J, Ramakrishna S (2005) Applications of polymer nanofibers in biomedicine and biotechnology. Appl Biochem Biotechnol 125: 147-158.

38. Krishnan R, Rajeswari R, Venugopal J, Sundarrajan S, Sridhar R, et al (2012) Polysaccharide nanofibrous scaffolds as a model for in vitro skin tissue regeneration. J Mater Sci Mater Med 23: 1511-1519.

39. Xu H, Su J, Sun J, Ren T (2012) Preparation and characterization of new nano-composite scaffolds loaded with vascular stents. Int J Mol Sci 13: 3366 3381.

40. Wang H, Feng Y, Fang Z, Xiao R, Yuan W, et al. (2012) Fabrication and Characterization of ElectrospunGelatin-Heparin Nanofibers as Vascular Tissue Engineering. Macromol Res 21: 860-869.

41. Vatankhah E, Prabhakaran MP, Semnani D, Razavi S, Morshed M, et al. (2014) Electrospun tecophilic/gelatin nanofibers with potential for smal diameter blood vessel tissue engineering. Biopolymers 101: 1165-1180.

42. Norouzi M, Shabani I, Ahvaz HH, Soleimani M (2015) PLGA/gelatin hybrid nanofibrous scaffolds encapsulating EGF for skin regeneration. J Biomed Mater Res A 103: 2225-2235.

43. Sundaramurthi D, Vasanthan KS, Kuppan P, Krishnan UM, Sethuraman $S$ (2012) Electrospun nanostructured chitosan-poly (vinyl alcohol) scaffolds: a biomimetic extracellular matrix as dermal substitute. Biomed. Mater 7 : 045005 .

44. Pezeshki-Modaress M, Mirzadeh H, Zandi M (2015) Gelatin-GAG electrospun nanofibrous scaffold for skin tissue engineering: fabrication and modeling of process parameters. Mater Sci Eng C Mater Biol Appl 48: 704-712.

45. Suganya S, Venugopal J, Ramakrishna S, Lakshmi BS, Giri Dev VR (2014) Aloe Vera/Silk Fibroin/Hydroxyapatite Incorporated ElectrospunNanofibrous Scaffold for Enhanced Osteogenesis. J Biomater Tissue eng 4: 9-19.
46. Srivastava S, Chakraborty A, Salunke R, Roy P (2012) Development of a Novel Polygalacturonic Acid-Gelatin Blend Scaffold Fabrication and Biocompatibility Studies for Tissue-Engineering Applications. Int J Polym Mater 61: 679-698.

47. Karuppuswamy P, Venugopal JR, Navaneethan B, Liava AL, Sridhar S, et al. (2014) Functionalized hybrid nanofibers to mimic native ECM for tissue engineering applications. Appl Surf Sci 322: 162-168.

48. Rho KS, Jeong L, Lee G, Seo BM, Park YJ, et al. (2006) Electrospinning of collagen nanofibers: effects on the behavior of normal human keratinocytes and early-stage wound healing. Biomaterials 27: 1452-1461.

49. Chong EJ, Phan TT, Lim IJ, Zhang YZ, Bay BH, et al. (2007) Evaluation of electrospun PCL/gelatin nanofibrous scaffold for wound healing and layered dermal reconstitution. Acta Biomater 3: 321-330.

50. Kim SE, Heo DN, Lee JB, Kim JR, Park SH, et al. (2009) Electrospun gelatin/ polyurethane blended nanofibers for wound healing. Biomed Mater 4: 044106

51. Gomes SR, Rodrigues G, Martins GG, Roberto MA, Mafra M, et al. (2015) In vitro and in vivo evaluation of electrospun nanofibers of PCL, chitosan and gelatin: a comparative study. Mater Sci Eng C Mater Biol Appl 46: 348-358.

52. Schneider A, Garlick JA, Egles C (2008) Self-assembling peptide nanofiber scaffolds accelerate wound healing. PLoS One 3: e1410.

53. Vashisth $P$, Singh H, Pruthi PA, Singh RP, Pruthi V (2014) Evaluation of gellan based electrospunnanofibers for wound healing. Proceedings of nanoconference 514-517.

54. Kim YJ, Bae HI, Kwon OK, Choi MS (2009) Three-dimensional gastric cancer cell culture using nanofiber scaffold for chemosensitivity test. Int $\mathrm{J}$ Biol Macromol 45: 65-71.

55. Moroni L, Schotel R, Hamann D, de Wijn JR, van Blitterswijk CA (2008) 3D Fiber-Deposited Electrospun Integrated Scaffolds Enhance Cartilage Tissue Formation. Adv Funct Mater 18: 53-60.

56. Cui W, Zhou $Y$, and Chang J (2010) Electrospunnanofibrous materials for tissue engineering and drug delivery. Sci Technol Adv Mater 11.

57. Kim K, Luu YK, Chang C, Fang D, Hsiao BS, et al. (2004) Incorporation and controlled release of a hydrophilic antibiotic using poly(lactide-co-glycolide)based electrospun nanofibrous scaffolds. J Control Release 98: 47-56.

58. Li C, Vepari C, Jin HJ, Kim HJ, Kaplan DL (2006) Electrospun silk-BMP-2 scaffolds for bone tissue engineering. Biomaterials 27: 3115-3124

59. Phipps MC, Xu Y, Bellis SL (2012) Delivery of Platelet-Derived Growth Facto as a Chemotactic Factor for Mesenchymal Stem Cells by Bone-Mimetic Electrospun Scaffolds. PLoS ONE 7: e40831.

60. Dang JM, Leong KW (2006) Natural polymers for gene delivery and tissue engineering. Adv Drug Deliv Rev 58: 487-499.

61. Lim SH, Liao IC, Leong KW (2006) Nonviral gene delivery from nonwoven fibrous scaffolds fabricated by interfacial complexation of polyelectrolytes. Mol Ther 13: 1163-1172.

62. Jannesari M, Varshosaz J, Morshed M, Zamani M (2011) Composite poly(viny alcohol)/poly(vinyl acetate) electrospun nanofibrous mats as a novel wound dressing matrix for controlled release of drugs. Int J Nanomedicine 6: 9931003.

63. Schneider A, Wang XY, Kaplan DL, Garlick JA, Egles C (2009) Biofunctionalized electrospun silk mats as a topical bioactive dressing for accelerated wound healing. Acta Biomater 5: 2570-2578.

64. Ma G, Liu Y, Peng C, Fang D, He B, et al. (2011) Paclitaxel loaded electrospun porous nanofiers as mat potential application for chemotherapy against prostate cancer. Carbohydr Polym 86: 505-512. 